\section{Miscellaneous: Poster Abstract}

\section{Case report of vaginal melanoma Inderjit Kaur \\ Rajiv Gandhi Cancer Institute and Research Centre, New Delhi, India}

Primary malignant melanoma of vagina is a rare disease with a predilection for local recurrence, distant metastasis and short survival time. Due to the low incidence and lack of reporting in the literature, treatment choices still remain controversial. We describe 2 cases of vaginal malignant melanoma. A $42 \mathrm{yr}$ old female presented with complaints of post coital and per vaginal bleed of 1 month duration. Examination findings shows growth $6 \mathrm{~cm} \times 6$ $\mathrm{cm}$ on anterior vaginal wall, another $3 \times 3 \mathrm{~cm}$ lesion on right lateral vagianl wall. Vaginal biopsy showed malignant melanoma, S-100 and HMB-45 positive while negative for CK and LCA. MRI Whole abdomen showed altered lesion [3.8 cm (AP), $6.0 \mathrm{~cm}(\mathrm{TR})$ and $4.9 \mathrm{~cm}(\mathrm{CC})]$ in upper $2 / 3^{\text {rd }}$ of vagina extending into vaginal fornices and abutting right lower cervix superiorly, right paravaginal extension and mesorectal fascia. No significant enlarged lymph nodes were seen. In view of localised disease she underwent Type III Radical hysterectomy with bilateral salpingo-ophorectomy with bilateral pelvic lymphnode dissection with total vaginectomy. Histopathology s/o 2 tumour nodules, one located in the anterior vaginal cuff measuring $-5 \times 5$ $\times 3.2 \mathrm{~cm}$, another located in right lateral vaginal cuff measuring $2.5 \times 3 \times 1.5$ $\mathrm{cm}$, malignant melanoma with involvement of the cervix with full thickness stromal invasion $(2.8 / 2.8 \mathrm{~cm}$,) invading perivaginal soft tissue, distance of invasive carcinoma from closest stromal margin $<0.1 \mathrm{~cm}$ (12 O' clock), LVI, $\mathrm{PNI}$ - not seen, all pelvic LN free (0/25). In view of positive margin and full thickness stromal involvement, she received radiotherapy to pelvis and Inguinal region to a dose of $45 \mathrm{~Gy} / 25 \#$ followed by a boost of $16 \mathrm{~Gy} / 8 \#$ to the tumour bed till $01 / 01 / 16$. Another case is a 40 yrs female, presented with complaints of bloody discharge per vaginum of 4 months duration. On examination, there was a large growth occupying the vagina till introitus. Cervix normal, para free. MRI Pelvis showed altered lesion involving left lateral uterine cervix and upper $2 / 3^{\text {rd }}$ of vagina with full thickness stromal involvement with mild left parametrial, anterior and posterior paravaginal extension, measuring $2.9 \times 4.5 \times 5.3 \mathrm{~cm}$. Few subcmlymphnodes were seen in bilateral external and internal iliac regions $(\mathrm{L}>\mathrm{R})$. Vaginal Biopsy was suggestive of Malignant Melanoma, expressing S-100, HMB 45 and SDX-10. Metastatic work up was negative. She underwent RH with total vaginectomy with bilateral PLND with RPLND. HPR showed exophytic black growth seen involving all quadrants of vagina, extending upwards into both lips of cervix $-7 \times 6 \times 2.5 \mathrm{~cm}$, Malignant melanoma, distance of invasive carcinoma from closest margin: $<0.1 \mathrm{~cm}$ (paravaginal soft tissue), $3 / 8$ right Pelvic LN, ECE + , 01/9 Left pelvic LN , ECE absent, 0/6 Right common iliac LN, 0/1 Reperitoneal $\mathrm{LN}$ was seen. She received adjuvant radiotherapy to a dose of $50 \mathrm{~Gy} / 25 \#$ to the pelvis and inguinals $\rightarrow$ boost of $6 \mathrm{~Gy} / 3 \#$ to nodal regions showing ECE $\& 10 \mathrm{~Gy} / 5 \#$ to the primary region.

\section{Miscellaneous: Poster Abstract}

\section{Case report of vaginal melanoma \\ Inderjit Kaur, Swarupa Mitra, Manoj Kumar Sharma, Upasna Saxena, Parveen Ahlawat, Amit Kumar Choudhary, Sarthak Tandon, Prashant Surkar}

Primary malignant melanoma of vagina is a rare disease with a predilection for local recurrence, distant metastasis and short survival time. Due to the low incidence and lack of reporting in the literature, treatment choices still remain controversial. We describe 2 cases of vaginal malignant melanoma. A $42 \mathrm{yr}$ old female presented with complaints of post coital and per vaginal bleed of 1 month duration. Examination findings show growth $6 \mathrm{~cm} \times 6 \mathrm{~cm}$ on anterior vaginal wall, another $3 \times 3 \mathrm{~cm}$ lesion on right lateral vaginal wall. Vaginal biopsy showed malignant melanoma, S-100 and HMB-45 positive while negative for CK and LCA. MRI Whole abdomen showed altered lesion [3.8cm (AP), $6.0 \mathrm{~cm}(\mathrm{TR})$ and $4.9 \mathrm{~cm}(\mathrm{CC})]$ in upper $2 / 3^{\text {rd }}$ of vagina extending into vaginal fornices and abutting right lower cervix superiorly, right paravaginal extension and mesorectal fascia. No significant enlarged lymph nodes were seen. In view of localised disease she underwent Type III Radical hysterectomy with bilateral salpingo-ophorectomy with bilateral pelvic lymphnode dissection with total vaginectomy. Histopathology s/o 2 tumour nodules, one located in the anterior vaginal cuff measuring $-5 \times 5 \times 3.2 \mathrm{~cm}$, another located in right lateral vaginal cuff measuring $2.5 \times 3 \times 1.5 \mathrm{~cm}$, malignant melanoma with involvement of the cervix with full thickness stromal invasion $(2.8 / 2.8$ $\mathrm{cm}$,) invading perivaginal soft tissue, distance of invasive carcinoma from closest stromal margin $<0.1 \mathrm{~cm}$ (12 O' clock), LVI, PNI - not seen, all pelvic $\mathrm{LN}$ free $(0 / 25)$. In view of positive margin and full thickness stromal involvement, she received radiotherapy to pelvis and Inguinal region to a dose of $45 \mathrm{~Gy} / 25$ \# followed by a boost of $16 \mathrm{~Gy} / 8$ \# to the tumour bed till $01 / 01 / 16$. Another case is a $40 \mathrm{yrs}$ female, presented with complaints of bloody discharge per vaginum of 4 months duration. On examination, there was a large growth occupying the vagina till introitus. Cervix normal, para free. MRI Pelvis showed altered lesion involving left lateral uterine cervix and upper $2 / 3^{\text {rd }}$ of vagina with full thickness stromal involvement with mild left parametrial, anterior and posterior paravaginal extension, measuring $2.9 \times 4.5 \times 5.3 \mathrm{~cm}$. Few subcmlymphnodes were seen in bilateral external and internal iliac regions ( $\mathrm{L}>\mathrm{R})$. Vaginal Biopsy was suggestive of Malignant Melanoma, expressing S-100, HMB 45 and SDX-10. Metastatic work up was negative. She underwent RH with total vaginectomy with bilateral PLND with RPLND. HPR showed exophytic black growth seen involving all quadrants of vagina, extending upwards into both lips of cervix $-7 \times 6 \times 2.5 \mathrm{~cm}$, Malignant melanoma, distance of invasive carcinoma from closest margin: $<0.1 \mathrm{~cm}$ (paravaginal soft tissue), 3/8 right Pelvic LN, ECE +, 01/9 Left pelvic LN , ECE absent, 0/6 Right common iliac LN, 0/1 Reperitoneal LN was seen. She received adjuvant radiotherapy to a dose of $50 \mathrm{~Gy} / 25$ \# to the pelvis and inguinals $\rightarrow$ boost of $6 \mathrm{~Gy} / 3$ \# to nodal regions showing ECE $\& 10 \mathrm{~Gy} / 5 \#$ to the primary region.

\section{Miscellaneous: Poster Abstract}

Extra ovarian adult granulosa cell tumor of omentum: A report of a rare entity

Anil Khurana, Paramjeet Kaur, Ashok K. Chauhan, Yashpal Verma, Nupur Bansal

Department of Radiotherapy, Pt. BDS PGIMS, Rohtak, Haryana, India

Aims: Extra ovarian granulosa cell tumor (GCT) is extremely rare tumor, assumed to arise from the ectopic gonadal tissue along the embryonal route of the genital ridge. A case of extra ovarian granulosa cell tumor of omentum in a 69 year old female presented here.

Materials and Methods: A 69 years old postmenopausal, hypertensive female presented with complaints of pain in right lumber and iliac region of one month duration. Pain was off and on and intermittent. The patient had a history of hysterectomy 12 years ago for fibroid uterus.

Results: Ultrasound examination of abdomen showed a hypoechoic lesion of size $78.1 \mathrm{~mm} \times 57.3 \mathrm{~mm}$ in right iliac fossa with mild thickening of surrounding omentum. Another hypoechoic lesion of size $36.7 \mathrm{~mm}$ x $22.9 \mathrm{~mm}$ was seen in retroperitoneal region in supero-medial aspect of right kidney. CECT abdomen showed heterogeneously enhanced nodular lesion of size $6.6 \mathrm{x}$ $6.8 \mathrm{~cm}$ in right lumbar region, mild thickening of surrounded omentum also seen however there was no evidence of infiltration to bowel loop seen. Uterus was not visualized. PET CT whole body revealed mildly metabolically active enlarged nodes in the bilateral level ib an ii, metabolically active large lobulated heterogeneously enhancing soft tissue density lesion in right lumbar region with non enhancing areas of necrosis. The lesion is closely abutting the anterior abdominal wall musculature antero laterally and small bowel loop medially surrounding mesenty shows increased vascularity and haziness. Colonoscopy findings were normal. Trucut biopsy of mass right lumbar region was positive for malignancy likely Round cell Sarcoma. A provisional diagnosis of retroperitoneal sarcoma of right lumbar region was made. She underwent exploratory laparotomy with excision of tumor. As per Operative findings there was approximately $8 \times 7 \mathrm{~cm}$, firm, omental mass present right to midline, arising from under surface of greater omentum. Ovaries were normal. Gross examination of omental mass showed nodular mass measuring $8 \times 5 \times 6 \mathrm{~cm}$. External surface was multinodular and cut surface was grey brown to grey yellow with solid cystic areas and areas of necrosis. Microscopic examination of specimen showed Extraovarian Adult granulosa cell tumor/metastasis from occult granulose cell tumor. On IHC Vimentin, CK, SMA, Inhibin were positive, Ki67:15\%, ER/PR were also positive and are negative for calretinin, thromobomodulin. Extensive necrosis was seen. After that she underwent rexploration and total omenectomy. HPE showed fat necrosis in omentum. 
All investigation showed no evidence of tumor in ovaries and at any other primary site then the patient finally diagnosed as having Granulosa cell tumor involving only omentum post op stage III C. Then patient was given six courses of chemotherapy with Inj Paclitaxel and Inj Carboplatin three weekly. Now patient is on regular follow up and disease free.

Conclusion: Extra ovarian adult granulosa cell tumor of omentum is rare tumor. Multimodal treatment approaches including surgery, multi-agent chemotherapy may provide a survival benefit for patients.

\section{Miscellaneous: Ovary Abstract}

\section{Synchronous malignancy of ovary and cervix Vani Malhotra, Smiti Nanda, Meenakshi Chauhan, Vandana Bhuria}

Background: Synchronous primary malignancies of the female genital tract constitute $1.7 \%$ of all genital malignancies.

Case: A 45-year-old para 5 woman presented with loss of appetite and abdominal distention. Provisional diagnosis of ovarian malignancy was made. Final histopathology of the specimen revealed ovarian papillary serous cystadenocacinoma with cervical leiomyosarcoma. She received chemotherapy. Results: Patient is on regular follow-up.

Conclusion: The coexistence of primary neoplasms in the ovary and cervix are rare. A normal appearing organ may have a hidden malignancy. So, every surgical specimen should be subjected to detailed histopathological examination. Also, the possibility of synchronous malignancy elsewhere in body should be kept in mind while working on a genital malignancy.

\section{Miscellaneous: Poster Abstract}

\section{Radical excision of a massive vulvo: Vaginal mass J. Meena, A. Parthasarathy, R. Vatsa, N. Singh, S. Kumar, K. K. Roy, S. Singhal \\ Department of Obstetrics and Gynaecology, AIIMS, New Delhi, India}

Background: Vulvo-vaginal masses has a varied presentation and causes. The most common differential diagnosis are Condylomata acuminata, Vulvular abscess, Vulvular/vaginal cysts, Vulval carcinoma, Vulval lipoma, Angiomyofibroblastoma and Aggressive Angiomyxoma. Surgical excision of the mass is the main method of treatment and the outcome differs with the histological diagnosis. We present a video of excision of a massive Vulvo vaginal mass in toto.

Case: A 45 year old P3 L3 female, presented with complaint of mass in perineal area $\&$ discharge per vaginum for 2 years. The mass was growing progressively and reached the present size. On examination there was a $9 \times 8 \mathrm{~cm}$ irregular firm to cystic mass, arising from posterior wall of vagina and protruding out of introitus with bossellated surface. The mass also extended into right ischiorectal fossa, $10 \times 10 \mathrm{~cm}$ mass with cystic, smooth surface that was irreducible with no cough impulse. CECT abdomen and pelvis revealed a well-defined $12 \times 10$ $\mathrm{X} 8 \mathrm{~cm}$ mass in right perineum arising from right lower lateral vaginal wall with ischiorectal fossa extension. There was no extension into cervix, bladder or rectum. Biopsy taken from the mass was inconclusive. A wide local excision was done under general anesthesia wherein an ischiorectal and vaginal mass of size $30 \times 10 \mathrm{~cm}$ with irregular margin was excised in toto. Histopathology was suggestive of Aggressive Angiomyxoma. The patient is under follow up. Discussion: Aggressive Angiomyxoma is a rare slow growing locally invasive mesenchymal tumor and has a substantial potential for recurrence. It is often misdiagnosed. Pre-operative diagnosis is difficult due to rarity of this entity and absence of diagnostic features, but it should be considered in case of masses in genital, perianal and pelvic region in a woman of reproductive age. Radical surgical excision is the first line of management. A long term follow up of the case is necessary and MRI is preferred method for detecting recurrences.

\section{Miscellaneous: Poster Abstract}

Distant pedicled musculocutaneous/fasciocutaneous flaps; a novel approach for reconstruction of large vulvar defects

Kripa Shanker Mishra

\section{RGCIRC, New Delhi, India}

Introduction: Postablative reconstruction of vulvar defects is a difficult challenge. Local flaps carry a high incidence of delayed wound healing as local flaps may redistribute but not eliminate local wound tension. Repair of defect with distant pedicled flaps may avert local complications by minimising tension to the skin and increasing the initial biomechanical strength of wound. The aim of this study was to determine the clinical outcome of distant musculocutaneous \& fasciocutaneous flaps used for postablative reconstruction of large vulvar defects.

Methods: Between January 2015 to December 2015 total three patients underwent vulvectomy and immediate reconstruction with distant pedicled flaps for vulvar carcinoma. Postoperative complications were recorded and clinical outcomes were evaluated.

Results: Two of the three flaps healed primarily. One flap was complicated by minor wound dehiscence, which healed with conservative treatment. Hospital stay and clinical course was shorter in comparison to local flaps and split skin graft.

Conclusions: Distant musculocutaneous and fasciocutaneous flaps provide excellent design flexibility and can be tailored to reconstruct postablative vulvar defects with good outcomes and minimal morbidity.

\section{Miscellaneous: Poster Abstract}

To find the prevalence of female genital tract malignancies in a tertiary care hospital

\section{Dilpreet K. Pandher}

Department of Obstetrics and Gynaecology, Government Medical College and Hospital, Sector 32, Chandigarh, India

Genital tract and breast are two most common sites of malignancy in females. Out of the genital tract malignancies, carcinoma cervix is so far found to be the commonest followed by ovary and endometrium. In developed countries, carcinoma cervix incidence is comparatively quite low due to good regular screening of females. One year review of patients was done, who underwent definitive/debulking surgery for a diagnosed malignant pathology of the genital tract, in obstetrics and gynaecology department of Govt medical College and Hospital, Chandigarh. Total 62 patients were operated, most common indication was carcinoma ovary, followed by endometrial cancer, cancer cervix and gestational trophoblastic neoplasia. 166 patients underwent biopsies for suspicious symptoms or the abnormal findings on examination and the patients with final malignancy report were either operated as described above and the inoperable cases were referred to oncotherapy department for further management.

\section{Miscellaneous: Poster Abstract}

Gestational trophoblastic neoplasia: Retrospective analysis of clinical profile, treatment pattern and outcome

Paramjeet Kaur, Ashok K. Chauhan, Anil Khurana, Yashpal Verma, Nupur Bansal

\section{Department of Radiotherapy, Pt. BDS PGIMS, Rohtak,} Haryana, India

Background: Gestational trophoblastic disease is a spectrum of cellular proliferation arising from the placental villous trophoblast. Gestational triphoblastic neoplasia (GTN) is a collective term for GTD that invade locally or metastasize. GTD includes hydatidiform mole (complete and partial) and GTN include invasive mole, choricocarcinoma, placental site trophoblastic tumor and epitheliod trophoblastic tumor.

Aim: To evaluate clinicopathological profile, treatment pattern and clinical outcome in patients with gestational trophoblastic neoplasia (GTN).

Materials and Methods: Twelve cases of gestational trophoblastic neoplasia treated between 2012 to November 2015 in deptt of Radiotherapy - II, PGIMS, Rohtak were evaluated in this retrospective study. Data was analyzed on the basis of age, histopathology, stage, type of treatment received and treatment related toxicities. Disease free survival was estimated.

Results: Out of 12 women 7 (58 \%) had hydatidiform mole, 4 (33\%) invasive mole and $01(8 \%)$ had choriocarcinoma. All the cases were given chemotherapy. Two patients had low risk disease. Among high risk group 